\title{
Ultrasound measurements of lumbar multifidus and abdominal muscle size in firefighters
}

\author{
James L. Nuzzo ${ }^{\mathrm{a}}$, Daniel W. Haun ${ }^{\mathrm{b}}$ and John M. Mayer ${ }^{\mathrm{a}, *}$ \\ ${ }^{a}$ School of Physical Therapy and Rehabilitation Sciences, Morsani College of Medicine, University of South \\ Florida, Tampa, FL, USA \\ ${ }^{\mathrm{b}}$ Department of Radiology, College of Chiropractic, Logan University, St. Louis, MO, USA
}

\begin{abstract}
.
BACKGROUND: Firefighters have a high incidence of low back pain and injury.

OBJECTIVE: To describe lumbar multifidus and abdominal muscle size characteristics in firefighters and to compare these characteristics to normative reference ranges.

METHODS: In career firefighters without current low back pain (62 males, 7 females), ultrasonography was used to assess: L4 and L5 lumbar multifidus cross-sectional area (CSA), asymmetry, and thicknesses; and external oblique, internal oblique, and transverse abdominal thicknesses. Comparisons of mean values in firefighters were made to the normative $95 \%$ reference ranges for the general population and soldiers.

RESULTS: Mean values for lumbar multifidus and abdominal muscle size in firefighters fell within the 95\% reference ranges for the general population and soldiers for all measurements, except L4 multifidus CSA in males and L5 multifidus CSA in females, which were larger in firefighters than the general population.

CONCLUSIONS: The majority of lumbar multifidus and abdominal muscle size measurements in firefighters are similar to the general population and soldiers. The larger lumbar multifidus CSA in firefighters compared with the general population warrants further study. These data provide a basis for future research using ultrasonography to assess muscle size in firefighters and other workers in physically-demanding occupations.
\end{abstract}

Keywords: Firefighters, muscles, spine, ultrasonography

\section{Introduction}

Firefighting is a dangerous occupation with a high incidence of low back injury and related pain syndromes [1]. In a recent study, 55\% of career firefighters reported current low back pain and $86 \%$ had a history of low back pain [1]. Back injury is also the leading cause of early retirement in firefighters [1].

${ }^{*}$ Corresponding author: John Mayer, School of Physical Therapy and Rehabilitation Sciences, Morsani College of Medicine, University of South Florida, 12901 Bruce B. Downs Blvd, MDC77; Tampa, Florida 33612, USA. Tel.: +1 813974 3818; Fax: +1 813974 8915; E-mail: lincolnchair@health.usf.edu.
The cause of low back pain and injury is often times multifactorial and firefighters are exposed to many of the common risk factors. For example, various physical activities performed by firefighters (e.g., operating charged hoses inside a building, climbing ladders, breaking windows, cutting structures, looking for hidden fires, and lifting objects $\geqslant 18 \mathrm{~kg}$ ) have been associated with low back injuries [2].

Lumbar multifidus and abdominal muscle size have been assessed with ultrasonography in various subject groups, and aberrations in lumbar multifidus and abdominal size and activation have been associated with low back pain [3]. Consequently, researchers have developed normative reference ranges for lumbar mul- 
tifidus and abdominal size to help identify potential muscle aberrations [4-6]. Despite the high incidence of low back pain in firefighters and the physically demanding firefighting work environment, no previous investigations have assessed lumbar multifidus and abdominal muscle size in firefighters. Therefore, no previous investigations have attempted to determine if firefighters fall within normative reference ranges for lumbar multifidus and abdominal muscle size. With firefighters at high risk of low back injury and pain, the identification of unique muscle size characteristics may have clinical implications. Furthermore, describing lumbar multifidus and abdominal muscle size in firefighters will extend the use of current normative reference ranges and provide additional comparative values for future studies that assess muscle size in firefighters with and without back pain. The purpose of this investigation was to describe lumbar multifidus and abdominal muscle size characteristics in firefighters and to compare these characteristics to normative reference ranges.

\section{Methods}

\subsection{Study design}

A cross-sectional study was conducted at a university laboratory. Data were collected during baseline assessments for a randomized controlled trial, which investigated the effect of progressive resistance exercise on back and core muscle endurance in firefighters (Grant EMW-2009-FP-00418 from the Federal Emergency Management Agency, United States Department of Homeland Security). The dependent variables were lumbar multifidus CSA, thickness, and asymmetry; and external oblique, internal oblique, and transverse abdominal thicknesses. Comparisons of the lumbar multifidus and abdominal muscle size measurements from firefighters in the current study were made to normative reference values that have been reported in the general population [4,5] and in soldiers [6].

\subsection{Subjects}

A convenience sample of career firefighters without current low back pain $(\mathrm{n}=69 ; 62$ males, 7 females), who were enrolled in a randomized controlled trial, participated in this study (Table 1). The average career length of the firefighters was $9.2 \pm 9.2$ (mean \pm standard deviation) years. Subjects were recruited
Table 1

Descriptive characteristics of firefighters in the current study

\begin{tabular}{lcl}
\hline Variable & Male & Female \\
\hline $\mathrm{n}$ & 62 & 7 \\
Age $(\mathrm{y})$ & $36.2 \pm 9.4$ & $25.1 \pm 3.6$ \\
Body height $(\mathrm{m})$ & $1.79 \pm 0.08$ & $1.66 \pm 0.05$ \\
Body mass $(\mathrm{kg})$ & $88.6 \pm 15.3$ & $67.1 \pm 9.5$ \\
BMI $\left(\mathrm{kg} / \mathrm{m}^{2}\right)$ & $27.7 \pm 3.7$ & $24.4 \pm 4.0$ \\
\hline
\end{tabular}

Values reported as mean \pm standard deviation. BMI $=$ body mass index.

from the entire population of firefighters $(n=573)$ of Tampa Fire Rescue (Tampa, Florida, United States), a medium-sized municipal fire department. Recruitment methods included presentations, email notices, posted information, and word-of-mouth. All candidates provided informed consent prior to participation in the onsite screening procedures. The experimental protocol complied with ethical standards and was approved by the university's institutional review board.

Screening of inclusion and exclusion criteria involved various self-reported health history questionnaires and a physical examination by a licensed physician. Inclusion criteria for participation were: 18 years of age or older and an active, full-duty firefighter. Exclusion criteria were: cardiovascular or orthopedic contraindications to exercise; history of systemic inflammatory disease or spinal surgery; clinically meaningful self-reported current low back pain or disability; level of readiness for physical activity at screening deemed to preclude participation; presence of a red flag for potential serious condition related to low back pain; abnormal resting blood pressure or heart rate; currently receiving care for spinal pain disorder/injury; currently diagnosed with or receiving care for a psychological or psychiatric disorder; currently performing progressive resistance exercises for the low back or core muscles; active workers' compensation or personal injury case; simultaneously enrolled in another clinical trial; drug or alcohol abuse within the past year; or any other condition, which in the opinion of the investigators would put the candidate at increased safety risk or otherwise make the candidate unsuitable for this study.

No participants enrolled in this study had current low back pain at the time of the ultrasound assessment of muscle size. Given the study's eligibility criteria, candidates with a history of clinically-meaningful low back pain were excluded from participation. Nevertheless, approximately $31.9 \%(22 / 69)$ had a lifetime history of any occurrence of self-reported low back pain or injury as determined by a "yes" response to the following question: "Have you ever injured your low back or had pain in your low back?" 


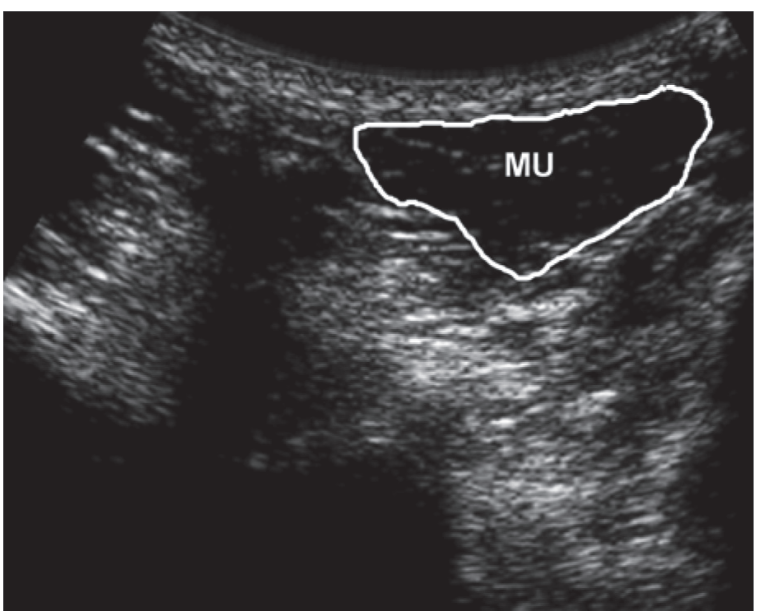

Fig. 1. Ultrasound image of L5 lumbar multifidus (MU) cross-sectional area.

\subsection{Ultrasonography}

Image acquisition: Muscle CSA and thickness images were acquired with a portable ultrasound device (MyLab 25, Biosound Esaote Inc., Florence, Italy) equipped with a $5 \mathrm{MHz}$ curvilinear transducer (CA631, Biosound Esaote Inc., Florence, Italy). Images of lumbar multifidus CSA were acquired from the subject's left and right sides at the L4 and L5 levels. Images of lumbar multifidus thickness were acquired from the subject's right side at the L4 and L5 levels. For all lumbar multifidus images, the subject assumed a prone position on a table, with a pillow underneath the abdomen as needed to standardize position of lumbar lordosis [7]. The position of the transducer for these images was determined by palpating bony landmarks and identifying these landmarks on the device's monitor.

Images of the external oblique, internal oblique, and transverse abdominal muscles were acquired with the subject resting in a supine position on a table, with a pillow underneath the head. These images were acquired from the subject's right side, with the transducer positioned along the midaxillary line, approximately half way between the iliac crest and the inferior angle of the rib cage [8]. The position of the transducer was then manipulated until all three muscles were visualized on the monitor with the anterior medial edge of the transverse abdominal approximately $2 \mathrm{~cm}$ from the edge of the image [8].

Image analysis: The images were stored on the hard drive of the ultrasound device in bitmap format. The images were then exported to a desktop computer, and then imported into ImageJ software (National Institute

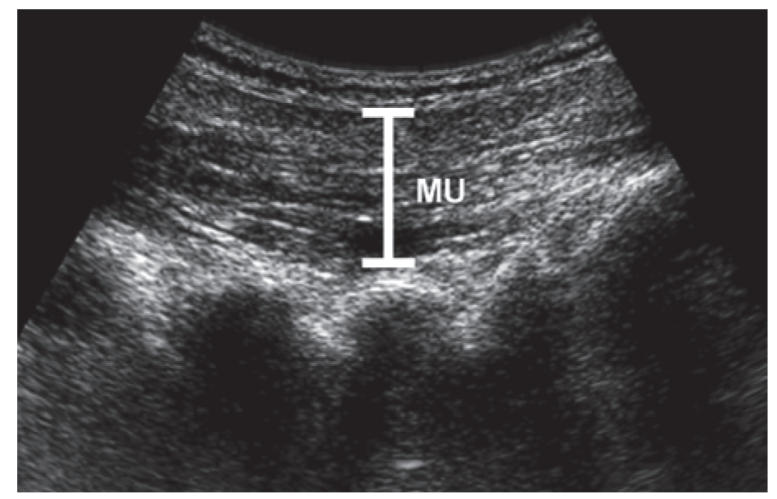

Fig. 2. Ultrasound image of L4 lumbar multifidus (MU) thickness.

of Health, Bethesda, Maryland, United States) for analysis. Anatomical landmarks were used in standardizing muscle CSA and thickness measurements. For lumbar multifidus CSA, the following landmarks were used: the echogenic lamina was used to identify the muscle's deep border, the acoustic shadow of the spinous process was used to identify the muscle's medial border, the thoracolumbar fascia was used to identify the muscle's superficial border, and the fascia separating the lumbar multifidus from the erector spinae was used to identify the muscle's lateral border. The freehand tool from ImageJ was then used to trace around the muscle's borders [7] (Fig. 1). Thickness of the lumbar multifidus muscle at the L4-L5 and L5-S1 facet joint levels was measured as the distance between the most superficial portion of the facet joint and the plane between the muscle and subcutaneous tissue [9] (Fig. 2). For the current study, these two variables were named L4 and L5 thickness. External oblique, internal oblique, and transverse abdominal muscle thicknesses were measured as the distances between the superficial and deep muscle fascias at the middle of the image [10] (Fig. 3). Pixel measurements of muscle thickness were exported from ImageJ into a spreadsheet and converted to linear measurements in centimeters. For all ultrasound variables, the mean value of two measurements from two separate images was used for further analysis. Ultrasonography has been shown to be a reliable and valid tool for assessing lumbar multifidus and abdominal muscle size $[9,11,12]$.

\subsection{Data analysis}

Data were analyzed using SPSS version 20 (IBM Corporation, Armonk, New York, United States). Descriptive statistics (mean \pm standard deviation) were used in assessing the CSA and thickness measure- 
Table 2

Lumbar multifidus and abdominal muscle size of firefighters in the current study

\begin{tabular}{lcr}
\hline Variable & Male & Female \\
\hline Cross-sectional area: & & $7.6 \pm 1.8(4.0-11.2)$ \\
L4 multifidus ( $\left.\mathrm{cm}^{2}\right)$ & $11.6 \pm 2.2(7.2-16.0)$ & $9.0 \pm 2.0(5.0-9.0)$ \\
L5 multifidus (cm $\left.{ }^{2}\right)$ & $11.5 \pm 2.1(7.3-15.7)$ & $13.7 \pm 8.6(0-30.9)$ \\
L4 multifidus side diff. (\%) & $9.2 \pm 8.8(0-26.8)$ & $4.8 \pm 3.3(0-11.4)$ \\
L5 multifidus side diff. (\%) & $9.8 \pm 15.1(0-40.0)$ & $2.4 \pm 0.2(2.0-2.8)$ \\
Thickness: & & $2.4 \pm 0.3(1.8-3.0)$ \\
L4 multifidus (cm) & $2.8 \pm 0.5(1.8-3.8)$ & $0.48 \pm 0.15(0.18-0.78)$ \\
L5 multifidus (cm) & $0.57 \pm 0.16(0.25-0.89)$ & $0.67 \pm 0.15(0.37-0.97)$ \\
Ext. obl. abdominal (cm) & $1.08 \pm 0.23(0.62-1.54)$ & $0.29 \pm 0.08(0.13-0.45)$ \\
Int. obl. abdominal (cm) & $0.41 \pm 0.12(0.17-0.65)$ & \\
Transverse abdominal (cm) & & \\
\hline
\end{tabular}

Values reported as mean \pm standard deviation (SD). The 95\% reference ranges are in parentheses, which were calculated as follows: $95 \%$ reference range $=$ mean $\pm 2 \mathrm{SD}$ [5]. Side diff $=$ Side difference calculated as [(largest side/smallest side) x 100] - 100) [5]. Ext. obl. = External oblique. Int. obl. = Internal oblique.

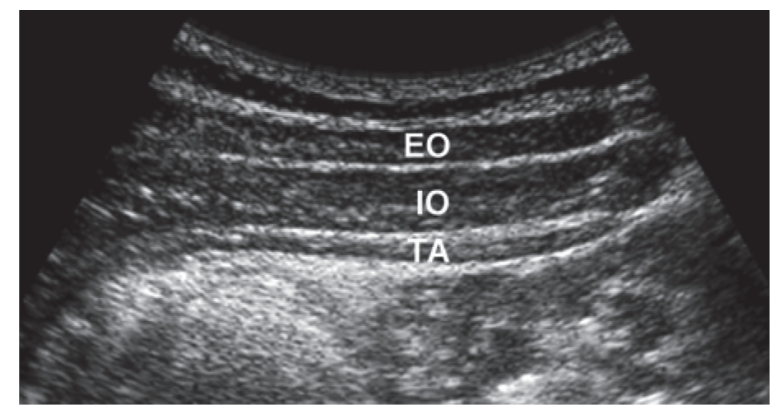

Fig. 3. Ultrasound image of external oblique (EO), internal oblique (IO), and transverse abdominal (TA) thicknesses.

ments in all subjects. Reference ranges (mean \pm 2 standard deviations) were calculated for the CSA and thickness measurements [5]. Asymmetry between right and left lumbar multifidus CSA was expressed as the percent difference between sides ( $\%$ difference $=$ [(largest/smallest value $) \times 100]-100)[5]$. Based on a previous suggestion that a $10 \%$ asymmetry of lumbar multifidus CSA may represent an abnormality [13], the number of firefighters exhibiting an asymmetry greater than or equal to $10 \%$ was tabulated. In addition, the muscle size characteristics of subjects with and without a history of low back pain were compared using independent $\mathrm{T}$ tests.

The muscle size measurements obtained in firefighters in the current study were compared with representative normative values as follows (specific comparisons were dependent on the availability of data reported in previous studies): Lumbar multifidus muscle size in firefighters was compared with the general population [5]. Lumbar multifidus muscle thickness in firefighters was compared with soldiers [6]. Abdominal muscle thickness in firefighters was compared with the general population [4] and soldiers [6].
Assumptions of normality were violated for some of the dependent variables (muscle size measurements) in the current study based on results from the ShapiroWilk test. Thus, t-tests could not be used to compare mean values from the current study to reference values reported in previous studies. Consequently, mean values from the current study were considered different from values reported in previous studies if the current study values fell outside of the $95 \%$ reference ranges (mean \pm 2 standard deviations) from the previous studies.

\section{Results}

Descriptive statistics for lumbar multifidus and abdominal size of firefighters in the current study are presented in Table 2. The number of male firefighters with an asymmetry greater than or equal to $10 \%$ was 21 $(34 \%)$ and $19(31 \%)$ at the L4 and L5 levels, respectively. The number of female firefighters with an asymmetry greater than or equal to $10 \%$ was $4(57 \%)$ and $1(14 \%)$ at the L4 and L5 levels, respectively. No differences $(p>0.05)$ in any of the lumbar multifidus and abdominal size measurements were noted between participants with and without a lifetime history of any occurrence of self-reported low back pain or injury.

Demographic characteristics or participants and descriptive statistics for lumbar multifidus and abdominal muscle size reported in previous studies with the general population [4,5] and soldiers [6] are presented in Tables 3 and 4, respectively. Mean values for lumbar multifidus and abdominal muscle size in firefighters fell within the $95 \%$ reference ranges from the general population and soldiers, with the exception of L4 multifidus CSA in males and L5 multifidus CSA in fe- 
Table 3

Descriptive characteristics of participants in previous studies that assessed lumbar multifidus and abdominal muscle size in the general population [4,5] and soldiers [6]

\begin{tabular}{|c|c|c|c|c|c|c|}
\hline \multirow[b]{2}{*}{ Variable } & \multicolumn{2}{|c|}{ Stokes et al. [5] } & \multicolumn{2}{|c|}{ Rankin et al. [4] } & \multicolumn{2}{|c|}{ Teyhen et al. [6] } \\
\hline & Male & Female & Male & Female & Male & Female \\
\hline $\mathrm{n}$ & 52 & 68 & 55 & 68 & 244 & 96 \\
\hline Age (y) & $40.1 \pm 13.0$ & $34.2 \pm 12.8$ & $40.6 \pm 14.1$ & $33.8 \pm 12.7$ & $21.8 \pm 3.90$ & $22.3 \pm 5.0$ \\
\hline Body height (m) & $1.78 \pm 0.06$ & $1.65 \pm 0.06$ & $1.80 \pm 0.07$ & $1.70 \pm 0.06$ & $1.77 \pm 0.08$ & $1.64 \pm 0.09$ \\
\hline Body mass (kg) & $82.8 \pm 11.0$ & $62.9 \pm 8.90$ & $82.5 \pm 11.9$ & $63.3 \pm 8.70$ & $78.6 \pm 10.6$ & $65.3 \pm 8.30$ \\
\hline $\operatorname{BMI}\left(\mathrm{kg} / \mathrm{m}^{2}\right)$ & $25.8 \pm 3.20$ & $23.0 \pm 3.10$ & $26.2 \pm 3.30$ & $23.2 \pm 3.10$ & $25.0 \pm 2.80$ & $24.5 \pm 2.90$ \\
\hline
\end{tabular}

Values reported as mean \pm standard deviation. BMI $=$ body mass index.

Table 4

Lumbar multifidus and abdominal muscle size reported in previous studies with the general population [4,5] and soldiers [6]

\begin{tabular}{|c|c|c|c|c|c|c|}
\hline \multirow[b]{2}{*}{ Variable } & \multicolumn{2}{|c|}{ Stokes et al. [5] } & \multicolumn{2}{|c|}{ Rankin et al. [4] } & \multicolumn{2}{|c|}{ Teyhen et al. [6] } \\
\hline & Male & Female & Male & Female & Male & Female \\
\hline \multicolumn{7}{|l|}{ Cross-sectional area: } \\
\hline L4 multifidus $\left(\mathrm{cm}^{2}\right)$ & $\begin{array}{c}7.9 \pm 1.9 \\
(4.2-11.5)\end{array}$ & $\begin{array}{l}5.6 \pm 1.3 \\
(3.0-8.1)\end{array}$ & NR & NR & NR & NR \\
\hline L5 multifidus $\left(\mathrm{cm}^{2}\right)$ & $\begin{array}{c}8.9 \pm 1.7 \\
(5.6-12.2)\end{array}$ & $\begin{array}{l}6.7 \pm 1.0 \\
(4.7-8.6)\end{array}$ & NR & NR & NR & NR \\
\hline L4 multifidus side diff. (\%) & $\begin{array}{c}9.6 \pm 8.1 \\
(0-25.9)\end{array}$ & $\begin{array}{l}7.2 \pm 7.0 \\
(0-21.2)\end{array}$ & NR & NR & NR & NR \\
\hline L5 multifidus side diff. (\%) & $\begin{array}{c}8.1 \pm 5.5 \\
(0-19.0)\end{array}$ & $\begin{array}{c}7.2 \pm 6.5 \\
(0-20.1)\end{array}$ & NR & NR & NR & NR \\
\hline \multicolumn{7}{|l|}{ Thickness: } \\
\hline L4 multifidus (cm) & NR & NR & NR & NR & $\begin{array}{l}3.1 \pm 0.5 \\
(2.1-4.1)\end{array}$ & $\begin{array}{l}2.7 \pm 0.4 \\
(1.9-3.5)\end{array}$ \\
\hline L5 multifidus (cm) & NR & NR & NR & NR & NR & NR \\
\hline Ext. obl. abdominal (cm) & NR & NR & $\begin{array}{c}0.67 \pm 0.17 \\
(0.33-1.01)\end{array}$ & $\begin{array}{c}0.59 \pm 0.18 \\
(0.23-0.95)\end{array}$ & $\begin{array}{c}0.80 \pm 0.19 \\
(0.42-1.18)\end{array}$ & $\begin{array}{c}0.67 \pm 0.16 \\
(0.35-0.99)\end{array}$ \\
\hline Int. obl. abdominal $(\mathrm{cm})$ & NR & NR & $\begin{array}{l}1.02 \pm 0.27 \\
(0.48-1.56)\end{array}$ & $\begin{array}{c}0.75 \pm 0.18 \\
(0.39-1.11)\end{array}$ & $\begin{array}{l}1.04 \pm 0.23 \\
(0.58-1.50)\end{array}$ & $\begin{array}{l}0.75 \pm 0.14 \\
(0.47-1.03)\end{array}$ \\
\hline Transverse abdominal $(\mathrm{cm})$ & NR & NR & $\begin{array}{c}0.54 \pm 0.11 \\
(0.32-0.76)\end{array}$ & $\begin{array}{c}0.39 \pm 0.08 \\
(0.23-0.55)\end{array}$ & $\begin{array}{c}0.39 \pm 0.09 \\
(0.21-0.57)\end{array}$ & $\begin{array}{c}0.33 \pm 0.09 \\
(0.15-0.51)\end{array}$ \\
\hline
\end{tabular}

Values reported as mean \pm standard deviation (SD). The 95\% reference ranges are in parentheses, which were calculated as follows: $95 \%$ reference range $=$ mean \pm 2 SD [5]. Side diff $=$ Side difference calculated as [(largest side/smallest side $) \times 100]-100)[5]$. Ext. obl. $=$ External oblique. Int. obl. = Internal oblique. $\mathrm{NR}=$ not reported.

males. Mean L4 multifidus CSA in male firefighters $\left(11.6 \mathrm{~cm}^{2}\right)$ was above (i.e. larger than) the $95 \%$ reference range reported in the general population (4.2$11.5 \mathrm{~cm}^{2}$ ). Mean L5 multifidus CSA in female firefighters $\left(9.0 \mathrm{~cm}^{2}\right)$ was above (i.e. larger than) the $95 \%$ reference range reported in the general population $\left(4.7-8.6 \mathrm{~cm}^{2}\right)$.

\section{Discussion}

This is the first study to describe lumbar multifidus and abdominal muscle size characteristics in firefighters. Although some variability in mean values was noted between firefighters and the reference populations, the majority of measurements were within the normative reference ranges. Similarities in lumbar multifidus and abdominal size between firefighters and other reference populations indicate that these measurements are fairly consistent across various healthy populations and that indications in one occupational group may be generalizable to other occupational groups. However, the observed difference in lumbar multifidus CSA between firefighters and the general population may limit this generalizability.

The current study found that L5 multifidus CSA in male firefighters and L4 multifidus CSA in female firefighters were similar to those reported in a normative general population [5], with the mean values falling within the $95 \%$ reference ranges reported in the general population. On the other hand, the L4 multifidus CSA in male firefighters and L5 multifidus CSA in female firefighters were larger (i.e. above the $95 \%$ normative reference range) than in the general population. While beyond the scope of this study, the relative hypertrophy of L4 multifidus CSA in male firefighters and L5 
multifidus CSA in female firefighters could be related to numerous factors, such as adaptive strategies related to chronic low back pain [14], adaptive strategies related to physical requirements of work, or the relatively larger body mass values in firefighters of the current study [15].

All lumbar multifidus CSA asymmetry measurements in firefighters were similar to those reported in a normative general population [5], with the mean values falling within the $95 \%$ reference ranges reported in the general population. Male firefighters in the current study exhibited an asymmetry of the lumbar multifidus CSA of approximately $9.5 \%$. This level of asymmetry is similar to what has been reported in the general population [5]. Hides et al. [13] have suggested that a $10 \%$ asymmetry of the lumbar multifidus muscle CSA is representative of a potential abnormality. While the mean level of lumbar multifidus asymmetry in the male firefighters was slightly below this criterion value, approximately one-third of the male firefighters had an asymmetry greater than or equal to $10 \%$. Similarly, a recent study discovered $10 \%$ asymmetry of the lumbar multifidus to be common in a sample of 126 males, who had no history of low back pain within the previous year of being assessed [16]. Since 10\% asymmetry of lumbar multifidus appears to be fairly common in samples without current low back pain, it may not be an appropriate criterion for abnormality. Asymmetry amongst firefighters and the general population may be a natural occurrence, or it may be related to the occupational and physical exercise training histories of the particular samples studied. Future studies that assess lumbar multifidus muscle size and pain over time may be helpful in clarifying the relationship between lumbar multifidus asymmetry and low back pain.

Another interesting finding of this study is that the lumbar multifidus CSA values at the L4 and L5 levels were nearly identical in male firefighters. This particular finding is unique because more cranial portions of the lumbar multifidus typically have smaller CSA [5, 17], and because the female firefighters did not demonstrate the same trend as their male counterparts. Reasons for why the male firefighters in this study exhibited similar multifidus CSA at the L4 and L5 vertebral levels are unclear. Future investigations with male firefighters of similar body builds will help to clarify if this is a natural characteristic exhibited in this group or a consequence of other potential factors (e.g., age, other anthropometric characteristics, occupational demands, physical activity background, and measurement error).

Lumbar multifidus thickness values were similar to those reported in a normative population of soldiers [6] and abdominal thickness values in firefighters were similar to those reported in a normative general population [4]. That is, all lumbar multifidus and abdominal thickness measurements for firefighters fell within the $95 \%$ reference ranges reported for the representative normative populations. Of the abdominal muscles, the transverse abdominal muscle has been implicated in low back pain syndromes [18]. Considering that the resting thickness of the transverse abdominal muscle in firefighters is similar to that reported in other representative normative populations without current low back pain, firefighters do not appear to exhibit any unique resting thickness characteristics of the transverse abdominal muscle that would implicate this parameter as a risk factor for future episodes of low back pain in firefighters.

In general, the firefighters in the current study demonstrated similar lumbar multifidus and abdominal muscle size characteristics as other reference populations for the majority of muscle size measures assessed. Thus, measurements of lumbar multifidus and abdominal muscle size appear to be similar across various healthy populations. These similarities may indicate that ultrasonography can be used in diagnosis and also in treatment follow-up of both firefighters and other similar populations with low back pain. These similarities also indicate that future studies, in which correlates with low back pain are identified in the general population and other occupational groups, may be generalizable to firefighters. In addition, the findings from the current study also appear to be consistent with findings from other health research in firefighters, in which firefighters are found to be similar to the general population [19]. However, two of the four measurements of lumbar multifidus CSA in firefighters were larger than those measurements reported in the general population. Thus, the practical applications and generalizability of the study's findings as described above may be limited.

A limitation to the current study was that only firefighters without current low back pain participated. Therefore, direct conclusions cannot be made about the relationship of low back pain and lower trunk muscle size in firefighters. Another limitation of the current study is that nearly one-third of participants had a lifetime history of any occurrence of self-reported low back pain or injury. Thus, whether the lumbar multifidus and abdominal muscle size values described for firefighters in the current study are fully representative of "healthy" firefighters is unclear. Also, given the study's cross-sectional design, the study did not corre- 
late muscle size measurements with future incidence of low back injury. Thus, future research is needed to determine if differences in lumbar multifidus and abdominal muscle size exist in firefighters with and without low back pain, and if muscle size measurements correlate with future incidence of low back pain in firefighters. Another limitation of the present study is that the sample size for female firefighters was small. While the percentage of female firefighters enrolled in the present study is reflective of the fire service in general, the results related to muscle size in female firefighters should be interpreted with caution given the small sample size.

In conclusion, this paper describes lumbar multifidus and abdominal muscle size characteristics in firefighters without low back pain and compares those characteristics to normative reference ranges. While the majority of lumbar multifidus and abdominal muscle size measurements in firefighters are similar to those reported in the general population and in soldiers, the observed difference in lumbar multifidus CSA between firefighters and the general population requires further study. The findings from the current study provide a basis for future researchers and clinicians using ultrasonography to assess lumbar multifidus and abdominal muscle size in firefighters and other workers at high risk for low back pain in order to assess for differences in presentation and also response to intervention.

\section{Acknowledgments}

This study was funded by a Fire Prevention and Safety Grant (Number: EMW-2009-FP-00418) from the Federal Emergency Management Agency, U.S. Department of Homeland Security. The authors wish to thank Tampa Fire Rescue for its assistance in organizing and implementing this study.

\section{References}

[1] International Association of Fire Fighters and International Association of Fire Chiefs. The Fire Service Joint Labor Management Wellness-Fitness Initiative, 3rd edition. Washington DC: International Association of Fire Fighters, 2008.

[2] Nuwayhid I, Stewart W, Johnson J. Work activities and the onset of first-time low back pain among New York City fire fighters. Am J Epidemiol. 1993; 137(5): 539-48.
[3] Koppenhaver SL, Hebert JJ, Parent EC, Fritz JM. Rehabilitative ultrasound imaging is a valid measure of trunk muscle size and activation during most isometric sub-maximal contrations: A systematic review. Australian J Phys. 2009; 55(3): 153-69.

[4] Rankin G, Stokes M, Newham DJ. Abdominal muscle size and symmetry in normal subjects. Muscle Nerve. 2006; 34(3): 320-6.

[5] Stokes M, Rankin G, Newham D. Ultrasound imaging of lumbar multifidus muscle: Normal reference ranges for measurements and practical guidance on the technique. Man Ther. 2005; 10: 116-26.

[6] Teyhen DS, Childs JD, Stokes MJ, Wright AC, Dugan JL, George SZ. Abdominal and lumbar muscle size and symmetry at rest and during contracted states normative reference ranges. J Ultrasound Med. 2012; 31(7): 1099-110.

[7] Hides JA, Stanton WR, McMahon S, Sims K, Richardson CA. Effect of stabilization training on multifidus muscle crosssectional area among young elite cricketers with low back pain. J Orthop Sports Phys Ther. 2008; 38(3): 101-8.

[8] Ferreira PH, Ferreira ML, Hodges PW. Changes in recruitment of the abdominal muscles in people with low back pain. Ultrasound measurement of muscle activity. Spine. 2004; 29 : 2560-6.

[9] Teyhen DS, George SZ, Dugan JL, Williamson J, Neilson BD, Childs JD. Inter-rater reliability of ultrasound imaging of the trunk musculature among novice raters. J Ultrasound Med. 2011; 30(3): 347-56.

[10] Hides JA, Wong I, Wilson SJ, Belavy DL, Richardson CA. Assessment of abdominal muscle function during a simulated unilateral weight-bearing task using ultrasound imaging. J Orthop Sports Phys Ther. 2007; 37(8): 467-71.

[11] Hides JA, Richardson CA, Jull GA. Magnetic resonance imaging and ultrasonsgraphy of the lumbar multifidus muscle. Spine. 1995; 20(1): 54-8.

[12] Koppenhaver SL, Hebert JJ, Fritz JM, Parent EC, Teyhen DS, Magel JS. Reliability of rehabilitative ultrasound imaging of the transversus abdominis and lumbar multifidus muscles. Arch Phys Med Rehabil. 2009; 90(1): 87-94.

[13] Hides JA, Gilmore C, Stanton WR, Bohlscheid E. Multifidus size and symmetry among chronic LBP and healthy asymptomatic subjects. Man Ther. 2008; 13(1): 43-9.

[14] Stokes MJ, Cooper RG, Morris G, Jayson MIV. Selective changes in multifidus dimensions in patients with chronic low back pain. Eur Spine J. 1992; 1: 38-42.

[15] Nuzzo JL, Mayer JM. Body mass normalisation for ultrasound measurements of lumbar multifidus and abdominal muscle size. Man Ther. 2013 June; 18(3): 237-42.

[16] Niemalainen R, Briand M, Battie MC. Substantial asymmetry in paraspinal muscle cross-sectional area in healthy adults questions its value as a marker of low back pain and pathology. Spine. 2011; 36(25): 2152-7.

[17] Sitilertpisan P, Hides JA, Stanton WR, Paungmali A, Pirunsan U. Multifidus muscle size and symmetry among elite weightlifters. Phys Ther Sport. 2012; 13(1): 11-5.

[18] Hodges PW. Is there a role for transversus abdominis in lumbo-pelvic stability? Man Ther. 1999; 4(2): 74-86.

[19] Drew-Nord DC, Hong O, Froelicher ES. Cardiovascular risk factors among career firefighters. AAOHN J. 2009; 57(10): 415-22. 